\title{
Some Metric Properties and a Constructive Task of a Semi-Regular 2n-Sides Polygon
}

\author{
Nenad Stojanovic \\ Department of Mathematics, Faculty of Agriculture, University of Banja Luka, Banja Luka, Bosnia and Herzegovina \\ Email address: \\ nenad.stojanovic@agro.unibl.org

\section{To cite this article:} \\ Nenad Stojanovic. Some Metric Properties and a Constructive Task of a Semi-Regular 2n-Sides Polygon. American Journal of Applied \\ Mathematics. Vol. 8, No. 4, 2020, pp. 176-181. doi: 10.11648/j.ajam.20200804.12
}

Received: May 13, 2020; Accepted: June 8, 2020; Published: June 17, 2020

\begin{abstract}
A simple polygon that either has equal all sides or all interior angles is called a semi-regular polygon. In terms of this definition, we can distinguish between two types of semi-regular polygons: equilateral polygons (that have equal all sides and different interior angles) and equiangular polygons (that have equal interior angles and different sides). To analyze the metric properties of semi-regular polygons, knowing only one basic element, e.g. the length of $a$ side, as in regular polygons, is not enough. Therefore, in addition to the side of $a$ semi-regular polygon, we use another characteristic element of it to analyze the metric features, and that is the angle $\delta=\angle(\mathrm{a}, \mathrm{b})$ between the side of $a$ semi-regular polygon $\mathscr{P}_{\mathrm{N}}$ and the side $b$ of its inscribed regular polygon $\mathcal{P}_{\mathrm{n}}$. Some metric properties of a semi-regular equilateral $2 \mathrm{n}$-sides polygon are analyzed in this paper with respect to these two characteristic elements. Some of the problems discussed in the paper are: convexity, calculation of surface area, dependence on the length of sides $a$ and $\delta$, calculation of the radius of the inscribed circle depending on the sides $a$ and angles $\delta$, and calculation of the surface area in which the radius of the inscribed circle is known, as well as the relationship between them. It has been shown that the formula for calculating the surface area of regular polygons results from the formula for the surface area of 2 n-side semi-regular, equilateral polygons. Further, by using these results, it has been shown that the cross-sections of regular polygons inscribed to semi-regular equilateral polygons, the vertices of equiangular semi-regular polygons, as well as the sides of the regular polygons inscribed to it, intersect in the same manner at the vertices of the equilateral semi-regular polygon. It has further been shown that the sides of the equiangular semi-regular polygon refer to each other as the sines of the angles created by the sides of the inscribed polygons and the side of the semi-regular polygon.
\end{abstract}

Keywords: Semi-Regular Polygons, Surface Ratio, Equilateral and Equiangular Semi-Regular Polygons

\section{Introduction}

A simple polygon $\mathcal{P}_{N} \equiv A_{1} A_{2} \ldots A_{N}$ that has equal all sides or equal all interior angles is called a semi-regular polygon $[8$, $9,10,11]$. In terms of definition, we distinguish between two types of semi-regular polygons: equiangular (having equal interior angles and different sides) and equilateral (having equal sides, and different interior angles). We consider that vertex $A_{i}, i=1,2, \ldots, N, i \in \mathbb{N}$ of a polygon is in an even position, or odd position, if index $i$ is even, or odd number, respectively. In this paper we consider convex equilateral semi-regular polygons. The marking is as follows:

1. $N$ is a number of sides in a semi-regular equilateral polygon,

2. $n$ is a number of sides in a regular polygon,
3. $a$ is a side in a semi-regular polygon $\mathcal{P}_{N}$,

4. $b_{j}, j \in \mathbb{N}$ side in a regular polygon $\mathcal{P}_{n}^{i}$ "inscribed" to a semi-regular polygon $\mathcal{P}_{N} \equiv A_{1} A_{2} \ldots A_{N}$, constructed by joining its vertices in even (or odd) positions,

5. Interior angles of a semi-regular polygon at odd vertices are marked with $\alpha$, and those at even vertices are marked with $\beta$

6. Isosceles triangles

$$
\triangle A_{1} A_{2} A_{3}, \triangle A_{3} A_{4} A_{5}, \ldots, \triangle A_{2 n-1} A_{2 n} A_{1}
$$

are triangles constructed over each side of an "inscribed" regular $n$-triangle.

7. $r$ is the radius of the inscribed circle to the semi-regular equilateral polygon (Figure 1).

In addition to these interpreted marks, all other marks will 
be interpreted when mentioned in a given definition.

To a semi-regular equilateral polygon $\mathcal{P}_{N} \equiv A_{1} A_{2} \ldots A_{N}$ with $N=2 \cdot n, n \geq 2, n \in \mathbb{N}$ and with equal sides there can be "inscribed" regular $n$-side polygons by joining odd vertices $\mathcal{P}_{n}^{1} \equiv A_{1} A_{3} A_{5} \ldots A_{2 n-3} A_{2 n-1}$, or even vertices $\mathcal{P}_{n}^{2} \equiv A_{2} A_{3} A_{4} \ldots A_{2 n-2} A_{2 n}$.

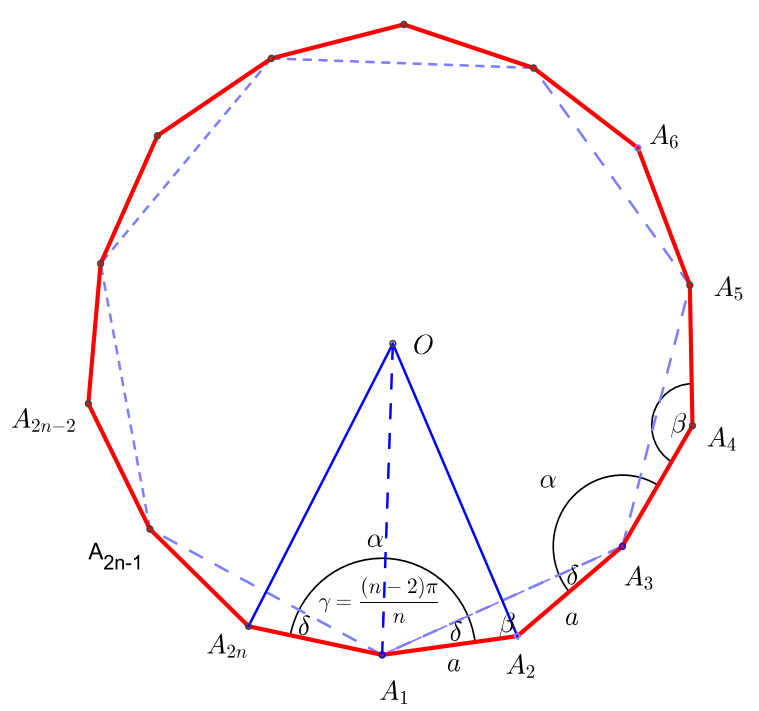

Figure 1. Basic elements of equilateral semi-regular polygon $\mathcal{P}_{2 n}^{a, \delta}$ of a side $a$ and angle $\delta$.

To analyze the metric properties of regular polygons, it is sufficient for us to know one basic element, i.e. the length of a side, while for the semi-regular polygons this is not sufficient [2].

Therefore, in addition to side $a$ of a semi-regular polygon, for the analysis of the metric properties we will use another element of it, and that is the angle between side $a$ of the semi-regular polygon and side $b$ of its "inscribed" regular polygon, which we mark with $\delta$, i.e. $\delta=\angle(a, b)$ (Figure 1) [3].

To show that a semi-regular equilateral $2 n-$ sides polygon is given by side $a$ and angle $\delta$, we write: $\mathcal{P}_{2 n}^{a, \delta}$.

If $\gamma=\frac{(n-2) \pi}{n}, n \geq 2$ is the interior angle of the "inscribed" regular polygon $\mathcal{P}_{n}^{1}$, then $\alpha=\gamma+2 \delta=\frac{(n-2) \pi}{n}+2 \delta$ gives interior angles at odd vertices, and $\beta=\pi-2 \delta$ gives the ones at even vertices of the semi-regular polygon $\mathcal{P}_{n}^{2}$ of a side $a$, where $\delta=\angle(b, a)$ marks the angle between the sides of polygons $\mathcal{P}_{n}^{1}$ and $\mathcal{P}_{2 n}$ (Figure 1). Here, we consider that a regular polygon with $n=2$ sides (segment) is "inscribed" to a semi-regular equilateral quadrilateral (rhombus).

Next, we consider those values of angle $\delta$ for which $\mathcal{P}_{N}$ is a convex semi-regular equilateral polygon. We find the values of angle $\delta$ for which semi-regular equilateral polygon $\mathcal{P}_{2 n}, n \geq 2, n \in \mathbb{N}$ is convex from the inequality which connects the definition of convexity with the values of interior angles of the semi-regular polygon [2].

That is, from inequality $\alpha<\pi$ i $\beta<\pi$ and values of angles $\alpha, \beta$ we find that for all $\delta \in\left\langle 0, \frac{\pi}{n}\right\rangle$ a semi-regular equilateral polygon $\mathcal{P}_{2 n}$ is convex, while for $\delta=\frac{2 \pi}{n}$ it is convex and regular. The following is true:

An equilateral semi-regular $2 n$-sides polygon of a side $a$ and angle $\delta$ is:

1. convex, if $\delta \in\left\langle 0, \frac{\pi}{n}\right\rangle$. For $\delta=\frac{\pi}{2 n}$ it is regular and convex.

2. non-convex, if $\delta \in\left\langle\frac{\pi}{n}, \frac{\pi}{2}\right\rangle$.

3. if $\delta>\frac{\pi}{2}$, then semi-regular polygon $\mathcal{P}_{2 n}$ is not defined [8-11].

\section{My Result}

\subsection{Surface of Semi-Regular Equilateral Polygon $\mathcal{P}_{2 n}^{a, \delta}$}

Let us show that the surface area of $\mathcal{P}(a, \delta)$ equilateral semi-regular $2 n$-sides polygon as a function of a side $a$ and angle $\delta$ is calculated by the formula (1).

Theorem 1. The surface area of equilateral semi-regular $2 \mathrm{n}$ - side polygon of side $a$ and angle $\delta$ is calculated by the of formula

$$
\mathcal{P}(a, \delta)=n a^{2} \frac{\cos \delta}{\sin \frac{\pi}{n}} \cos \left(\frac{\pi}{n}-\delta\right),
$$

where $n$ is the number of sides of the "inscribed" regular polygon $n \geq 2, n \in \mathbb{N}$.

Proof: Note that for the surface area of the inscribed equilateral semi-regular polygon $\mathcal{P}_{2 n}^{a, \delta}$ the following equality is valid

$$
\mathcal{P}(a, \delta)=\mathcal{A}\left(P_{n}^{b}\right)+n \mathcal{A}\left(P_{3}\right)
$$

where $\mathcal{A}\left(P_{n}^{b}\right)$ is the surface area of the inscribed regular $n$ sides polygon of a side $b$, and $\mathcal{A}\left(P_{3}\right)$ is the surface area of the isosceles triangle.

Let us further note a fragment of a semi-regular polygon (Figure 2). It follows from a special right triangle $\triangle A_{1} A_{2} K$ that $b=2 a \cos \delta$, so the surface area of an isosceles triangle is $\mathcal{A}\left(P_{3}\right)=a^{2} \sin \delta \cos \delta$.

Further, for the surface area of the "inscribed" polygon $\mathcal{P}_{n}$, according to the markings in Figure 2, the following is valid

$$
\mathcal{A}\left(P_{n}^{b}\right)=\frac{n}{4} b^{2} \cot \frac{\alpha}{2}=\frac{n}{4}(2 a \cos \delta)^{2} \cot \frac{\pi}{n}=n a^{2} \cos ^{2} \delta \cot \frac{\pi}{n} .
$$

Based on that, the surface area of a semi-regular equilateral polygon $\mathcal{P}_{2 n}$ is

$$
\begin{gathered}
\mathcal{P}(a, \delta)=n a^{2} \cos ^{2} \delta \cot \frac{\pi}{n}+n a^{2} \sin \delta \cos \delta \\
=n a^{2} \cos \delta\left(\cos \delta \cot \frac{\pi}{n}+\sin \delta\right)=\ldots=n a^{2} \frac{\cos \delta}{\sin \frac{\pi}{n}} \cos \left(\frac{\pi}{n}-\delta\right) .
\end{gathered}
$$

Corollary 2. Notice that for $\delta=\frac{\pi}{2 n} 2 n$-sides polygon becomes a regular polygon and the formula for calculating the surface area is

$$
\mathcal{P}(a, \delta)=n a^{2} \frac{\cos \frac{\pi}{2 n}}{\sin \frac{\pi}{n}} \cos \left(\frac{\pi}{n}-\frac{\pi}{2 n}\right)
$$




$$
\begin{aligned}
& =n a^{2} \frac{\cos \frac{\pi}{2 n}}{2 \sin \frac{\pi}{2 n} \cos \frac{\pi}{2 n}} \cos \frac{\pi}{2 n} \\
& =\frac{n a^{2}}{2} \cot \frac{\pi}{2 n}=\frac{(2 n) a^{2}}{4} \cot \frac{\pi}{2 n} .
\end{aligned}
$$

The latter equality is a formula for calculating the surface area of a regular $2 n$-sides polygon.

If in the formula for calculating the surface area of a semiregular polygon we include that $n=2$, then we get that

$$
\begin{gathered}
\mathcal{P}(a, \delta)=2 \cdot a^{2} \cdot \frac{\cos \delta}{\sin \frac{\pi}{2}} \cdot \cos \left(\frac{\pi}{2}-\delta\right) \\
=2 a^{2} \cos \delta \cos \left(\frac{\pi}{2}-\delta\right) \\
=a^{2} \sin (2 \delta),
\end{gathered}
$$

i.e. we get a formula for calculating the surface area of an equilateral quadrilateral (rhombus), where $\delta$ is the angle between the diagonal and the side of the rhombus.

\subsection{Radius of the Inscribed Circle}

For a semi-regular equilateral convex $2 n$-sides polygon of a side $a$ and angle $\delta$ to which the circle of radius $r$ can be inscribed, the following theorem holds:

Theorem 3. The radius of a circle inscribed to a semiregular equilateral polygon $\mathcal{P}_{2 n}^{a, \delta}$ depending on a side a and angle $\delta$ is calculated by the following formula

$$
r=a \frac{\cos \delta \cos \left(\frac{\pi}{n}-\delta\right)}{\sin \frac{\pi}{n}}
$$

where $n$ is the number of sides of the "inscribed" regular polygon and $n \geq 2$ and $n \in \mathbb{N}$.

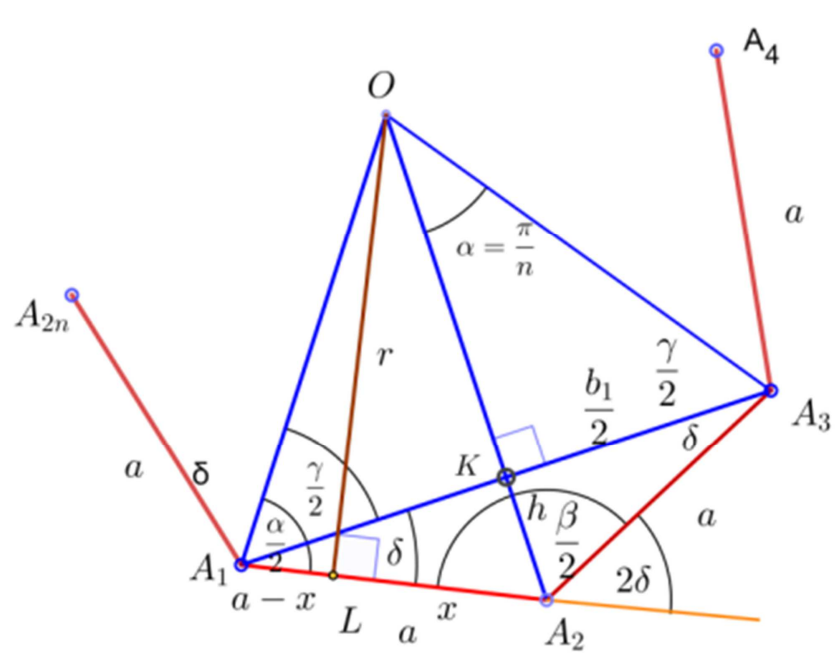

Figure 2. A fragment of $2 n$-sides polygon.

Proof: From a special right triangle $\triangle O A_{1} L$ (Figure 2) we have that $\tan \frac{\alpha}{2}=\frac{r}{a-x}$ and from it $x=a-\frac{r}{\tan \frac{\alpha}{2}}$. Similarly, from a special right triangle $\Delta O A_{2} L$ it follows that $\tan \frac{\beta}{2}=\frac{r}{x}$.
If we include the calculated value for $x$ in the latter equality we find that

$$
r=\left(a-\frac{r}{\tan \frac{\alpha}{2}}\right) \tan \left(\frac{\pi}{2}-\delta\right) .
$$

If we express radius $r$ from this equality, after calculation, we find that

$$
r=\frac{a \cdot \tan \frac{\beta}{2}}{1+\frac{\tan \frac{\beta}{2}}{\tan \frac{\alpha}{2}}}
$$

Since,

$$
\begin{gathered}
\frac{\alpha}{2}=\frac{(n-2) \pi}{2 n}+\delta \text { and } \frac{\beta}{2} \\
=\frac{\pi}{2}-\delta \text { and } \tan \left(\frac{\pi}{2}-\frac{\pi}{n}\right)=\cot \frac{\pi}{n},
\end{gathered}
$$

from the latter equality, after replacement, we find that the radius of the inscribed circle can be obtained by the formula

$$
r=a \frac{\cot \delta \cot \left(\frac{\pi}{n}-\delta\right)}{\cot \delta+\cot \left(\frac{\pi}{n}-\delta\right)}
$$

From this equality, we obtain the required form

$$
r=a \frac{\cos \delta \cos \left(\frac{\pi}{n}-\delta\right)}{\sin \frac{\pi}{n}}
$$

Corollary 4. If in the latter equality we put that $=\frac{\pi}{n}$, we get that $r=\operatorname{acot} \frac{\pi}{n}$, i.e. the radius of the inscribed circle of a regular $\mathcal{P}_{2 n}$ polygon.

Corollary 5. If we write equality

$$
r=a \frac{\cos \delta \cos \left(\frac{\pi}{n}-\delta\right)}{\sin \frac{\pi}{n}} \text { in a form of } \frac{r}{a}=\frac{\cos \delta \cos \left(\frac{\pi}{n}-\delta\right)}{\sin \frac{\pi}{n}}
$$

and substitute it in the formula for the surface area of a semiregular equilateral $2 n-$ sides polygon, after calcuation we get the following:

$$
\mathcal{P}(a, \delta)=n a^{2} \frac{r}{a}=n \cdot a \cdot r .
$$

From this it follows that the surface area of a semi-regular equilateral polygon $\mathcal{P}_{2 n}$ is equal to the product of the length of the side, the radius of the inscribed circle, and the number of sides of the inscribed regular $n$-sides polygon.

Also, if in equality $r=a \frac{\cos \delta \cos \left(\frac{\pi}{n}-\delta\right)}{\sin \frac{\pi}{n}}$ we put that $a=$ $\frac{b}{2 \cos \delta}$, after calculation we find that the radius of the inscribed circle to a semi-regular equilateral polygon depending on a side $b$ of a regular polygon $\mathcal{P}_{n}{ }^{1}$ inscribed to it and angle $\delta=\angle(a, b)$, can be expressed by the following relation

$$
r=\frac{b}{2} \frac{\cos \left(\frac{\pi}{n}-\delta\right)}{\sin \frac{\pi}{n}} .
$$


Proposition 5. The surface area of a semi-regular equilateral polygon with $N=2 n, n \geq 2, n \in \mathbb{N}$, sides can be calculated by the following formula

$$
\mathcal{P}_{2 n}=\frac{n b r}{2 \cos \delta}
$$

where $b$ is the side of the inscribed regular $n$-sides polygon, $r$ is the radius of the inscribed circle and $\delta=\angle(a, b)$ is the angle between the side of the semi-regular polygon and the inscribed semi-regular $n$-sides polygon.

Proof. Since $\frac{2 r}{b}=\frac{\cos \left(\frac{\pi}{n}-\delta\right)}{\sin \frac{\pi}{n}}$ from the formula for the surface area of a semi-regular $2 n$-sides polygon

$$
\mathcal{P}_{2 n}=n a^{2} \frac{\cos \delta}{\sin \frac{\pi}{n}} \cos \left(\frac{\pi}{n}-\delta\right)
$$

and based on the previous relations we have that

$$
\begin{aligned}
\mathcal{P}_{2 n} & =n a^{2} \frac{\cos \delta}{\sin \frac{\pi}{n}} \cos \left(\frac{\pi}{n}-\delta\right)=n a^{2} \cos \delta \frac{2 r}{b} \\
& =n \cos \delta \frac{2 r}{b} \frac{b^{2}}{4 \cos ^{2} \delta}=\frac{n r b}{2 \cos \delta} .
\end{aligned}
$$

\subsection{A Constructive Task}

Let us formulate and prove the following theorem, which is related to the problem of a semi-regular $2 n$-sides polygon and regular polygons inscribed therein.

Theorem 6. Let $\mathcal{A}_{2 n} \equiv A_{1} A_{2} \ldots A_{2 n-1} A_{2 n}, n>2$ be a convex equilateral semi-regular $2 \mathrm{n}$-sides polygon and $\mathcal{P}_{n}^{1} \equiv A_{1} A_{3} A_{5} \ldots A_{2 n-3} A_{2 n-1}, \mathcal{P}_{n}^{2}=A_{2} A_{3} A_{4} \ldots A_{2 n-2} A_{2 n}$ be regular n-sides polygons inscribed therein, formed by joining the vertices at odd or even positions. To be proven:

1. the intersection points of the sides of these regular polygons are the vertices of an equiangular semi-regular $2 n$-sides polygon $\mathcal{B}_{2 n} \equiv B_{1} B_{2} B_{3} \ldots B_{2 n-1} B_{2 n}$,

2. the lengths of the sides of that equiangular polygon $\mathcal{B}_{2 n}$ relate as the sine of the angles closed by the sides of regular polygons with the side of a semi-regular equilateral 2 n-sides polygon $\mathcal{A}_{2 n}$.

Proof. a) Let there be a fragment of a semi-regular equilateral $2 n$-sides polygon

$$
\begin{gathered}
\mathcal{A}_{2 n} \equiv A_{1} A_{2} \ldots A_{2 n} \text { of a side } a \text { and } \\
\mathcal{P}_{n}^{1} \equiv A_{1} A_{3} A_{5} \ldots A_{2 n-3} A_{2 n-1} \\
\mathcal{P}_{n}^{2} \equiv A_{2} A_{3} A_{4} \ldots A_{2 n-2} A_{2 n}
\end{gathered}
$$

and let here be a regular $n$-sides polygon of sides $b_{1}$, $b_{2}$ respectively, inscribed to it, and let $\delta=\Varangle\left(a, b_{1}\right)$ be the angle between a side $a$ and a side $b_{1}$ of a regular n-sides polygon $\mathcal{P}_{n}^{1} \quad$ Let $\quad B_{1} B_{2}=c_{1}, B_{2} B_{3}=c_{2}, \ldots, B_{2 n-1} B_{2 n}=$ $c_{1}, B_{2 n} B_{1}=c_{2}$ be sides of a 2 n-sides polygon $\mathcal{B}_{2 n} \equiv$ $B_{1} B_{2} B_{3} \ldots B_{2 n-1} B_{2 n}$ constructed by the intersection of the sides of regular polygons $\mathcal{P}_{n}^{1}$ and $\mathcal{P}_{n}^{2}$ (Figure 3 ).

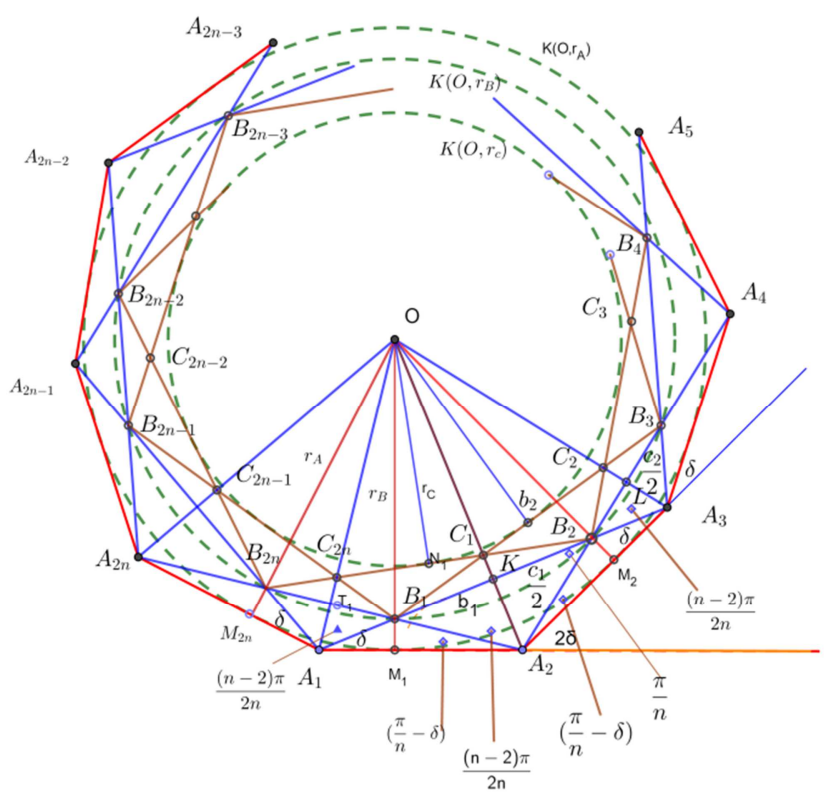

Figure 3. Fragment of a semi-regular $2 n$-sides polygon with basic elements.

Let us first prove that a constructed 2 n-sides polygon $\mathcal{B}_{2 n} \equiv B_{1} B_{2} B_{3} \ldots B_{2 n-1} B_{2 n}$ has equal all interior angles. Note that the following is valid for the interior angles of a semi-regular equilateral polygon $\mathcal{A}_{2 n}$ at vertices in odd positions,

$$
\begin{aligned}
& \measuredangle A_{1}=\measuredangle A_{3}=\cdots \\
= & \measuredangle A_{2 n-3}=\measuredangle A_{2 n-1} \\
= & \alpha=\frac{(n-2) \pi}{n}+2 \delta
\end{aligned}
$$

and for the interior angles at vertices in even positions, the following is valid

$$
\begin{gathered}
\measuredangle A_{2}=\measuredangle A_{4}=\cdots \\
=\measuredangle A_{2 n-2}=\measuredangle A_{2 n} \\
=\beta=\frac{(n-2) \pi}{n}+2\left(\frac{\pi}{n}-\delta\right)=\pi-2 \delta
\end{gathered}
$$

Since

$$
A_{1} A_{2}=A_{2} A_{3}=\ldots=a_{2 n-1} A_{2 n}=A_{2 n} A_{1}=a
$$$$
\text { and } \measuredangle A_{1}=\measuredangle A_{3}=\cdots=\measuredangle A_{2 n-3}=\measuredangle A_{2 n-1}=\delta \quad \text { and }
$$
$\measuredangle A_{2}=\measuredangle A_{4}=\cdots=\Varangle A_{2 n-2}=\Varangle A_{2 n}=\frac{\pi}{n}-\delta \quad, \quad$ triangles $\triangle A_{1} B_{1} A_{2}, \triangle A_{2} B_{2} A_{3,}, \Delta A_{2 n} B_{2 n} A_{1}$ are congruent, and, hence their interior angles are also congruent, i.e. the following is valid:

$\Varangle A_{1} B_{1} A_{2} \cong \Varangle A_{2} B_{2} A_{3} \cong \cdots$

$$
\cong \Varangle A_{2 n} B_{2 n} A_{1}=\frac{(n-1) \pi}{n}
$$

Note that the interior angles of a 2 n-sides polygon $\mathcal{B}_{2 n} \equiv B_{1} B_{2} B_{3} \ldots B_{2 n-1} B_{2 n}$ are cross-sectional to angles in (11), therefore, they are congruent with them, and it follows that

$$
\measuredangle B_{i}=\frac{(n-1) \pi}{n}, i=1,2, \ldots, 2 n, n \in \mathbb{N}
$$


It follows from (12) that a 2 n-sides polygon $\mathcal{B}_{2 n} \equiv$ $B_{1} B_{2} B_{3} \ldots B_{2 n-1} B_{2 n}$ is equiangular. Let us prove that an equiangular 2 n-sides polygon

$\mathcal{B}_{2 n} \equiv B_{1} B_{2} B_{3} \ldots B_{2 n-1} B_{2 n}$ has different sides. Note that triangles $\quad \Delta B_{1} A_{2} B_{2}, \Delta B_{3} A_{4} B_{4}, \cdots \Delta B_{2 n-1} A_{2 n} B_{2 n}, \Delta$ $B_{2 n-1} A_{1} B_{1}$ are congruent with each other because of the following:

$\Varangle B_{1}=\Varangle B_{2}=\cdots=\Varangle B_{2 n-1}=\Varangle B_{2 n}=\frac{\pi}{n}$ and $\measuredangle A_{2}=$ $\measuredangle A_{4}=\cdots=\measuredangle A_{2 n-2}=\measuredangle A_{2 n}=\frac{(n-2) \pi}{n}$, and from the congruency of triangles

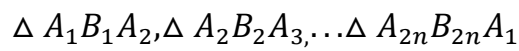

the congruency of the sides follows. Similarly, we conclude that triangles

$\triangle B_{2} A_{3} B_{3}, \Delta B_{4} A_{5} B_{5,} \ldots \Delta B_{2 n-2} A_{2 n-1} B_{2 n-1}, \Delta B_{2 n} A_{1} B_{1}$ are also mutually congruent because $\measuredangle B_{2}=\Varangle B_{3}=\cdots=$ $\measuredangle B_{2 n-1}=\measuredangle B_{2 n}=\frac{\pi}{n} \quad$ and $\quad \measuredangle A_{1}=\measuredangle A_{3}=\cdots=\measuredangle A_{2 n-1}=$ $\frac{(n-2) \pi}{n}$, and from the congruency of triangles $\triangle A_{1} B_{1} A_{2}, \Delta$

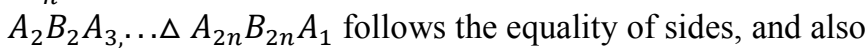
the diversity of their bases

$$
B_{1} B_{2}=c_{1}, B_{2} B_{3}=c_{2}, \ldots, B_{2 n-1} B_{2 n}=c_{1},
$$

$B_{2 n} B_{1}=c_{2}$ because these triangles are not isosceles. In doing so, we have proved that a 2 n-sides polygon $\mathcal{B}_{2 n} \equiv$ $B_{1} B_{2} B_{3} \ldots B_{2 n-1} B_{2 n}$ is an equiangular semi-regular polygon.

b) In general, observe a triangle $\triangle A_{1} A_{2} A_{3}$ with the given marks (Figure 3). For the interior angles of a triangle it is true that $\measuredangle A_{1}=\measuredangle A_{3}=\delta, \measuredangle A_{2}=\frac{(n-2) \pi}{n}+2\left(\frac{\pi}{n}-\delta\right)$ and that $A_{1} A_{2} B_{1}=\frac{\pi}{n}-\delta$. From the special right angle triangle $\triangle A_{1} K A_{2}$ we find that the side of the inscribed $n$-sides polygon is $\mathcal{P}_{n}^{1}, b_{1}=2 a \cos \delta$, and from the special right angle triangle $\triangle A_{2} K B_{1}$ we find that $\measuredangle A_{2}=\frac{(n-2) \pi}{n}$ and $\measuredangle B=\frac{\pi}{n}$, as well as that one side of a $2 n$-sides polygon $-\mathcal{B}_{2 n}$ is given in a relation

$$
c_{1}=\frac{2 a \sin \delta}{\tan \frac{\pi}{n}}, n>2, n \in \mathbb{N}
$$

because for $n=2$ we have a rhombus to which it is not possible to inscribe a semi-regular equiangular polygon.

From equiangular triangle $\triangle B_{2} L A_{3}$ we find that the side of the inscribed regular $\mathcal{P}_{n}^{2}$ is given in a relation

$$
b_{2}=2 a \cdot \cos \left(\frac{\pi}{n}-\delta\right)
$$

and that the other side of equiangular $2 n$-sides polygon $\mathcal{B}_{2 n}$ is given in a relation

$$
c_{2}=\frac{2 \operatorname{asin}\left(\frac{\pi}{n}-\delta\right)}{\tan \frac{\pi}{n}}, n>2, n \in \mathbb{N}
$$

Based on relations (13) and (15), we find that

$$
\frac{c_{2}}{c_{1}}=\frac{\frac{2 \operatorname{asin}\left(\frac{\pi}{n}-\delta\right)}{\tan \frac{\pi}{n}}}{\frac{2 a \sin \delta}{\tan \frac{\pi}{n}}}=\frac{\sin \left(\frac{\pi}{n}-\delta\right)}{\sin \delta} \mathbf{\square} .
$$

On the right-hand side of this formula (16), note the function $f(n, \delta)=\frac{\sin \left(\frac{\pi}{n}-\delta\right)}{\sin \delta}$ and select the value of angle $\delta$ such that it is possible to construct it geometrically, e.g. in the form of $\delta=\frac{\pi}{2^{n}}$.

Note that from the Bernoulli's inequality $(1+x)^{n} \geq 1+$ $n x$, (which for $x=1$, transforms into a form of $2^{n} \geq 1+$ $n>n$ ), it follows that inequalities $\frac{\pi}{2^{n}} \leq \frac{\pi}{n+1}<\frac{\pi}{n}$ are valid for every natural number $n \in \mathbb{N}$, and thus inequalities

$$
\frac{\pi}{2^{n}}=\frac{\pi}{2 \cdot 2^{n-1}}=\frac{\frac{\pi}{2^{n-1}}}{2}<\frac{\pi}{2^{n-1}} \leq \frac{\pi}{n} \text { are also valid for } n \in \mathbb{N} \text {. }
$$

Therefore, let the value of angle $\delta$ be given by relation $\delta(n)=\frac{\pi}{2^{n-1}} \in\left\langle 0, \frac{\pi}{n}\right\rangle$ for $n>2, n \in \mathbb{N}$ then function $f(n, \delta)$ takes the form of:

$$
f(n)=\frac{\sin \left(\frac{\pi}{n}-\frac{\pi}{2^{n-1}}\right)}{\sin \frac{\pi}{2^{n-1}}}, n \in \mathbb{N} .
$$

Note that for $n=1$ a function $f(1)$ is not defined, and for $\mathrm{n}=2$, it is $f(2)=\frac{\sin (0)}{\sin \frac{\pi}{2}}=0$, which is of no interest here given the nature of the function.

Example: For $n=3$ it is $\delta(3)=\frac{\pi}{4}$ and

$$
\begin{gathered}
f(3)=\frac{\sin \left(\frac{\pi}{3}-\frac{\pi}{2^{2}}\right)}{\sin \frac{\pi}{2^{2}}}=\frac{\sin \left(\frac{\pi}{3}-\frac{\pi}{4}\right)}{\sin \frac{\pi}{4}}=\frac{\sin \frac{\pi}{12}}{\sin \frac{\pi}{4}}=\sqrt{2} \sin \frac{\pi}{12}=\sqrt{2} . \\
\frac{1}{2} \sqrt{2-\sqrt{3}}=\frac{1}{2} \sqrt{4-2 \sqrt{3}} .
\end{gathered}
$$

From relation (16), it follows that the ratio of the sides of a semi-regular equiangular hexagon inscribed to a semi-regular equilateral hexagon is $\frac{c_{2}}{c_{1}}=\frac{1}{2} \sqrt{4-2 \sqrt{3}}$. For $n=4$ it is $\delta(4)=\frac{\pi}{8}$, and the value of the function is

$$
f(4)=\frac{\sin \left(\frac{\pi}{4}-\frac{\pi}{2^{4-1}}\right)}{\sin \frac{\pi}{2^{4-1}}}=\frac{\sin \frac{\pi}{8}}{\sin \frac{\pi}{8}}=1,
$$

so $\frac{c_{2}}{c_{1}}=1 \Rightarrow c_{2}=c_{1}$. So it follows that $\mathcal{B}_{2 n}^{c_{1}, c_{2}}$ and $\mathcal{A}_{2 n}^{a, \delta}$ are regular polygons with internal angles of $\frac{3 \pi}{4}$, that is, the following proposition is valid:

Proposition 7. For the value of angle $\delta(n)$ expressed in a formula: $\delta(n)=\frac{\pi}{2^{n-1}}, n>2, n \in \mathbb{N}$ semi-regular equiangular polygon $\mathcal{B}_{2 n}^{c_{1}, c_{2}}$ is regular, if and only if $n=4$.

Proof: Let us suppose that equiangular $2 n$-sides polygon $\mathcal{B}_{2 n}^{c_{1}, c_{2}}$ is a regular polygon. Then it is also true that $c_{1}=c_{2}$. Further from the requirement $f(n)=1$ we have the following equality $\frac{\sin \left(\frac{\pi}{n}-\frac{\pi}{2^{n-1}}\right)}{\sin \frac{\pi}{2^{n-1}}}=1$ which, after calculation, becomes: $\quad 2 \cos \frac{\pi}{2^{n}} \cdot \sin \left(\frac{\pi}{n}-\frac{\pi}{2^{n-1}}\right)=0 \Leftrightarrow \sin \left(\frac{\pi}{n}-\frac{\pi}{2^{n-1}}\right)=$ $0 \Leftrightarrow 2^{n-1}=2 n$.

In the set of natural numbers, only solution $n=4$ meets the requirement $n>2$.

\section{Conclusion}

Based on the content presented in this paper, it follows that 
the basic metric properties, such as: the convexity, the surface area of a semi-regular $2 \mathrm{n}$-sides polygon, the radius of the inscribed circle and the ratios of a semi-regular $2 n$-sides polygon can be expressed by side $a$ and angle $\delta$.

\section{References}

[1] D. G. Ball, The constructability of regular and equilateral poligons on square pinboard, Math. Gaz, V57, 1973, pp. $119-122$.

[2] A. A. Egorov, Rešetki i pravilnie mnogougolniki, Kvant $\mathrm{N}_{0} 12$, 1974, pp. 26-33.

[3] M. Audin, Geometry, Springer, Heidelberg, 2002.

[4] M. Polonio, D. Crnokvić, T. B. Kirigan, Z. Franušić, R. Sušanj, Euklidski prostori, PMF, Zagreb, 2008, pp. 51-57.

[5] Kirilov, O pravilnih mnogougolnikah, funkciji Eulera i ćisla Ferma, Kvant, $\mathrm{N}_{0}$ 6, 1994.

[6] M. Panov, A. Spivak, Vpisanie poligoni, Kvant, $\mathrm{N}_{0} 1,1999$.

[7] M. Radojčić, Elementarna Geometrija, Naučna knjiga, Beograd, 1961.

[8] N. Stojanović, Some metric properties of general semi-regular polygons, Global Journal of Advanced Research on Classical and Modern Geometries, Vol. 1, Issue 2, 2012, pp. 39-56.

[9] N. Stojanović, Inscribed circle of general semi-regular polygon and some of its features, International Journal of Geometry, Vol. 2., 2013, $\mathrm{N}_{0}$. 1, pp. 5-22.

[10] N. Stojanović, V. Govedarica, Jedan pristup analizi konveksnosti i računanju površine jednakostranih polupravillnih poligona, II MKRS, Zbornik radova, Trebinje, 2013, pp. 87-105.

[11] N. Stojanović, Neka metrička svojstva polupravilnih poligona, Filozofski fakultet Pale, 2015, disertacija.

[12] N. Stojanović, V. Govedarica, Diofantove jednačine i parketiranje ravni polupravilnim poligonima jedne vrste, Fourth mathematical conference of the Republic of Srpska, Proceedings, Volume I, Trebinje, 2015, pp. 183-194.

[13] N. Stojanović, V. Govedarica, Diofantove jednačine i parketiranje ravni polupravilnim poligonima dvije vrste, Sixth mathematical conference of the Republic of Srpska, Proceedings, Pale, 2017, pp. 266-280.

[14] V. V. Vavilov, V. A. Ustinov, Okružnost na rešetkah, Kvant, $\mathrm{N}_{0}$ 6, 2007.

[15] V. V. Vavilov, V. A. Ustinov, Mnogougolniki na rešetkah, Izdavateljstvo, MCIMO, Moskva, (2006). 